\title{
Enforcing Performance Isolation Across Virtual Machines in Xen
}

\author{
Diwaker Gupta ${ }^{1}$, Ludmila Cherkasova ${ }^{2}$, Rob Gardner $^{2}$, and Amin Vahdat ${ }^{1}$ \\ ${ }^{1}$ University of California, San Diego, CA 92122, USA \\ \{dgupta, vahdat\}acs.ucsd.edu \\ ${ }^{2}$ Hewlett-Packard Laboratories \\ \{lucy. cherkasova, rob.gardner\}@hp.com
}

\begin{abstract}
Virtual machines (VMs) have recently emerged as the basis for allocating resources in enterprise settings and hosting centers. One benefit of VMs in these environments is the ability to multiplex several operating systems on hardware based on dynamically changing system characteristics. However, such multiplexing must often be done while observing per-VM performance guarantees or service level agreements. Thus, one important requirement in this environment is effective performance isolation among VMs. In this paper, we address performance isolation across virtual machines in Xen [1]. For instance, while Xen can allocate fixed shares of CPU among competing VMs, it does not currently account for work done on behalf of individual VMs in device drivers. Thus, the behavior of one VM can negatively impact resources available to other VMs even if appropriate per-VM resource limits are in place.

In this paper, we present the design and evaluation of a set of primitives implemented in Xen to address this issue. First, XenMon accurately measures per-VM resource consumption, including work done on behalf of a particular VM in Xen's driver domains. Next, our $S E D F-D C$ scheduler accounts for aggregate VM resource consumption in allocating CPU. Finally, ShareGuard limits the total amount of resources consumed in privileged and driver domains based on administrator-specified limits. Our performance evaluation indicates that our mechanisms effectively enforce performance isolation for a variety of workloads and configurations.
\end{abstract}

\section{Introduction}

Virtual Machine Monitors (VMMs 1 are gaining popularity for building more agile and dynamic hardware/software infrastructures. In large enterprises for example, VMMs enable server and application consolidation in emerging on-demand utility computing models [2]3]. Virtualization holds the promise of achieving greater system utilization while lowering total cost of ownership and responding more effectively to changing business conditions.

Virtual machines enable fault isolation- "encapsulating" different applications in self-contained execution environments so that a failure in one virtual machine does not

\footnotetext{
${ }^{1}$ We use the terms hypervisor and domain interchangeably with VMM and VM respectively.
} 
affect other VMs hosted on the same physical hardware. performance isolation is another important goal. Individual VMs are often configured with performance guarantees and expectations, e.g., based on service level agreements. Thus, the resource consumption of one virtual machine should not impact the promised guarantees to other VMs on the same hardware.

In this paper, we focus on performance isolation mechanisms in Xen [1], a popular open source VMM. Xen supports per-VM CPU allocation mechanisms. However, it like many other VMMs - does not accurately account for resource consumption in the hypervisor on behalf of individual VMs, e.g., for I/O processing. Xen's I/O model has evolved considerably over time. In the initial design [1] shown in Figure 1a, the Xen hypervisor itself contained device driver code and provided shared device access. To reduce the risk of device driver failure/misbehavior and to address problems of dependability, maintainability, and manageability of I/O devices, Xen moved to the architecture shown in Figure 1b [4]. Here, "isolated driver domains" (IDDs) host unmodified (legacy OS code) device drivers. Domain-0 is a privileged control domain used to manage other domains and resource allocation policies.

This new I/O model results in a more complex CPU usage model. For I/O intensive applications, CPU usage has two components: CPU consumed by the guest domain, where the application resides, and CPU consumed by the IDD that incorporates the device driver and performs I/O processing on behalf of the guest domain. However, the work done for I/O processing in an IDD is not charged to the initiating domain. Consider a guest domain limited to $30 \%$ CPU consumption. If the work done on its behalf within an IDD to perform packet processing consumes $20 \%$ of the CPU, then that domain may consume $50 \%$ of overall CPU resources. Such unaccounted CPU overhead is significant for I/O intensive applications, reaching 20\%-45\% for a web server [5].

The key contribution of this paper is the design of a set of cooperating mechanisms to effectively control total CPU consumption across virtual machines in Xen. There are a number of requirements for such a system. First, we must accurately measure the resources consumed within individual guest domains. Next, we must attribute the CPU consumption within IDDs to the appropriate guest domain. The VMM scheduler must be modified to incorporate the aggregate resource consumption in the guest domain and work done on its behalf in IDDs. Finally, we must limit the total amount of work done on behalf of a particular domain in IDDs based on past consumption history and target resource limits. For instance, if a particular domain is already consuming nearly its full

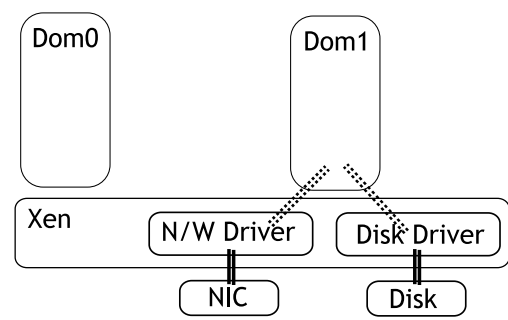

(a) I/O Model in Xen 1.0

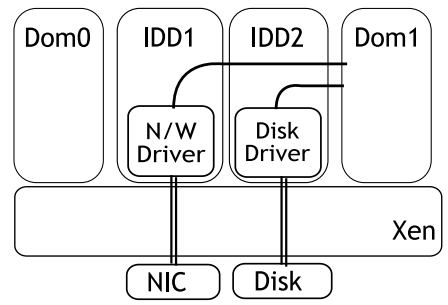

(b) I/O Model in Xen 3.0

Fig. 1. Evolution of Xen's I/O Architecture 
resource limits, then the amount of resources available to it in the IDDs must be scaled appropriately.

The analog of accounting resources consumed on behalf of a guest domain have come up in scheduling operating system resources across individual tasks 6 6 7 8 9 9 |10 11 12], e.g., in accounting for resources consumed in the kernel on behalf of individual processes. Our work builds upon these earlier efforts, exploring the key challenges associated with constructing appropriate abstractions and mechanisms in the context of modern VM architectures. One of the interesting problems in this space is developing minimally intrusive mechanisms that can: i) account for significant asynchrony in the hypervisor and OS and ii) generalize to a variety of individual operating systems and device drivers (performance isolation will quickly become ineffective if even a relatively small number of devices or operations are unaccounted for). To this end, we have completed a full implementation and detailed performance evaluation of the necessary system components to enable effective VM performance isolation:

- XenMon: a performance monitoring and profiling tool that reports (among other things) CPU usage of different VMs at programmable time scales. XenMon includes mechanisms to measure CPU for network processing in net-IDDs (IDDs responsible for network devices) on behalf of guest domains.

- SEDF-DC: a new VM scheduler with feedback that effectively allocates CPU among competing domains while accounting for consumption both within the domain and in net-IDDs.

- ShareGuard: a control mechanism that enforces a specified limit on CPU time consumed by a net-IDD on behalf of a particular guest domain.

\section{XenMon}

To support resource allocation and management, we implemented an accurate monitoring and performance profiling infrastructure, called XenMon 2 There are three main components in XenMon (Figure 2):

- xentrace: This is a lightweight event logging facility present in Xen. XenTrace can log events at arbitrary control points in the hypervisor. Each event can have some associated attributes (for instance, for a "domain scheduled" event, the associated attributes might be the ID of the scheduled domain and the event's time stamp). Events are logged into "trace buffers": shared memory pages that can be read by user-level Domain-0 tools. Note that xentrace was already implemented in Xen - our contribution here was to determine the right set of events to monitor.

- xenbaked: The events generated by XenTrace are not very useful on their own. xenbaked is a user-space process that polls 3 the trace buffers for new events and processes them into meaningful information. For instance, we collate domain sleep and wake events to determine the time for which a domain was blocked in a given interval.

- xenmon: This is the front-end for displaying and logging the data.

\footnotetext{
${ }^{2}$ Our implementation of XenMon has been integrated into the official Xen 3.0 code base.

${ }^{3}$ In the current implementation, events are posted via a virtual interrupt instead of periodic polling.
} 


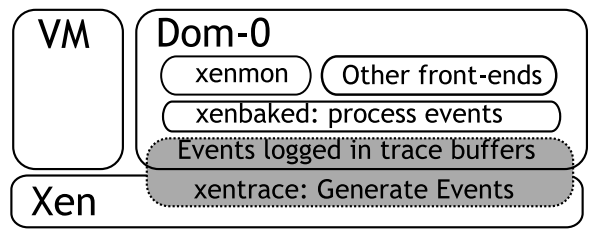

Fig. 2. XenMon Architecture

XenMon aggregates a variety of metrics across all VMs periodically (configurable with a default of $100 \mathrm{~ms}$ ). For this paper, we only use the CPU utilization and network accounting facilities (Section 3) of XenMon. Details on all the metrics available from XenMon and some examples of using XenMon for analyzing CPU schedulers in Xen are available separately [13].

\section{Network I/O Accounting}

Recall that one of the challenges posed by the new I/O model in Xen is to classify IDD CPU consumption across guest domains. This work is focused on network I/O, so we summarize network I/O processing in Xen. As mentioned earlier, in the IDD model a designated driver domain is responsible for each hardware device and all guests wishing to use the device have to share it via the corresponding IDD. The IDD has a "back-end" driver that multiplexes I/O for multiple "front-end" drivers in guest VMs over the real device driver. Figure 3 shows this I/O architecture in more detail. Note that for the experiments reported in this paper, we use Domain- 0 as the driver domain.

We briefly describe the sequence of events involved in receiving a packet - the numbers correspond to those marked in Figure 3 When the hardware receives the packet (1), it raises an interrupt trapped by Xen (2). Xen then determines the domain responsible for the device and posts a virtual interrupt to the corresponding driver domain via the event channel (3). When the driver domain is scheduled next, it sees a pending interrupt and invokes the appropriate interrupt handler. The interrupt handler in the driver domain only serves to remove the packet from the real device driver (4) and hand it over to the "back-end" driver (5), netback in Figure 3. Note that no TCP/IP protocol processing is involved in this step (except perhaps the inspection of the IP header).
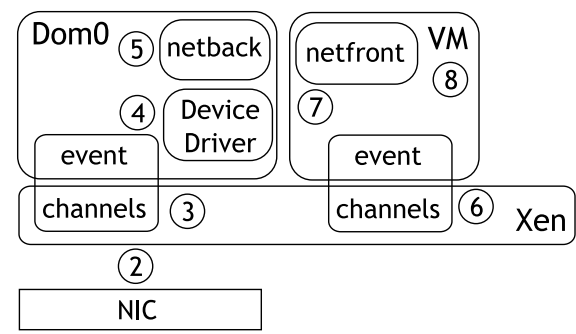

(1)

Fig. 3. I/O processing path in Xen 
It is netback's responsibility to forward the packet to the correct "front-end" driver (netfront in Figure 3). The driver domain transfers the ownership of the memory page containing the packet to the target guest domain, and then notifies it with a "virtual interrupt" (6). Note that this involves no data movement/copying. When the target guest is next scheduled, it will field the pending interrupt (7). The netfront driver in the guest will then pass on the packet to higher layers of the networking stack for further processing (8). The transmit path of a packet is similar, except that no explicit memory page exchange is involved (see [1] for details).

Thus, I/O processing in a net-IDD primarily involves two components: the real device driver and the back-end (virtual) device driver. One natural approach for more accurate accounting is to instrument these components for detailed measurements of all the delays on the I/O path. However, this approach does not scale in Xen for two reasons: (1) since Xen uses legacy Linux drivers, this would require instrumenting all network device drivers, and (2) network drivers involve significant asynchronous processing, making it difficult to isolate the time consumed in the driver in the context of a given operation.

We therefore need some alternate heuristics to estimate the per-guest CPU consumption. Intuitively, each guest should be charged in proportion to the amount of I/O operations it generates. In [5], we used the number of memory page exchanges as an estimator. However, we found this method to be a rather coarse approximation that does not take into account what fraction of these page exchanges correspond to sent versus received packets, and that does not take into account the size of the packets.

Thus, we propose using the number of packets sent/received per guest domain for distributing the net-IDD CPU consumption among guests. Note that netback is an ideal observation point: all of the packets (both on the send and receive paths between driver domain and guest domain) must pass through it. We instrumented netback to provide detailed measurements on the number of packets processed by the corresponding net-IDD in both directions for each guest domain. In particular, we added XenTrace events for each packet transmission/reception, with the appropriate guest domain as an attribute. We then extended XenMon to report this information.

Of course, knowing the number of packets sent and received on a per-domain basis does not by itself enable accurate CPU isolation. We need a mechanism to map these values to per-domain CPU consumption in the IDD. In particular, we want to know the dependence of packet size on CPU processing overhead and the breakdown of send versus receive packet processing. To answer these questions, we perform the following two part study.

The Impact of Packet Size on CPU Overhead in Net-IDD: We performed controlled experiments involving sending packets of different sizes at a fixed rate to a guest VM. In particular, we fixed the rate at $10,000 \mathrm{pkts} / \mathrm{sec}$ and varied the packet size from 100 to 1200 bytes. Each run lasted 20 seconds and we averaged the results over 10 runs. We repeated the experiments to exercise the reverse I/O path as well - so the VM was sending packets instead of receiving them. To prevent "pollution" of results due to ACKS going in the opposite direction, we wrote a custom tool for these benchmarks using UDP instead of TCP. The other end point for these experiments was a separate 


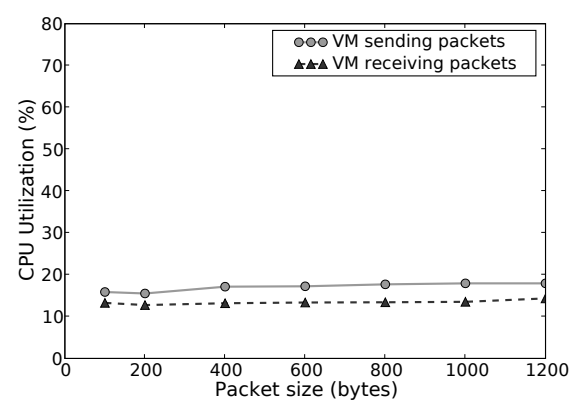

Fig. 4. CPU overhead in Domain-0 for processing packets at a fixed rate under different packet sizes

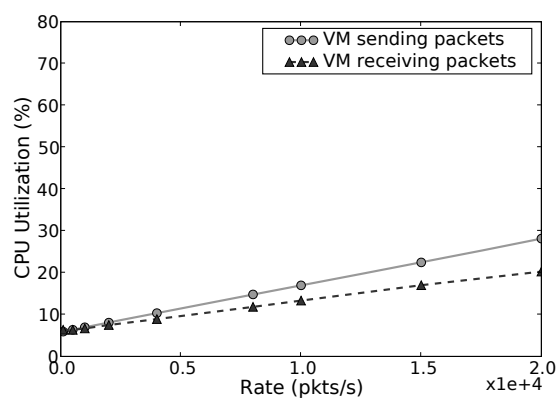

Fig. 5. CPU overhead in Domain-0 for processing packets of a fixed size under varying rates

machine on our LAN. Recall that in all of our experiments, we use Domain-0 to host the network device driver.

Our results show that CPU consumption in net-IDD does not depend on packet size as presented in Figure 4 The explanation is as follows: during driver packet processing there is no payload processing or copying; the driver largely deals with the packet header. For the rest of the I/O path within the net-IDD, there is no data copying (where CPU processing can depend on packet size) — only the ownership of memory pages changes to reflect data transfer.

CPU Overhead in Net-IDD for Send vs. Receive I/O Paths: In this experiment, we fixed the packet size at 256 bytes and varied the rate at which a VM sends or receives packets. We could thus selectively exercise the send and receive I/O paths within Xen and measure the resulting CPU overhead in net-IDD. We denote these as Send Benchmark and Receive Benchmark, respectively. As before, each run lasted 20 seconds and we averaged results over 10 runs.

Figure 5 presents our experimental results. An interesting outcome of this study is that the ratio of CPU consumption in net-IDD between send and receive paths is consistently the same for different packet rates. We denote this measured ratio as weight.

To validate the generality of presented results we repeated all of the experiments presented above for two different hardware configurations: a single CPU Intel Pentium-IV machine running at 2.66-GHz with a 1-Gbit Intel NIC (SYSTEM-1) and a dual processor Intel Xen 2.8-GHz with a 1-Gbit Broadcom NIC (SYSTEM-2). For both systems under test, the CPU consumption in net-IDD does not depend on packet size. Further, for both system under test, the ratio of CPU consumption in net-IDD between send and receive paths is consistent for different packet rates:

- SYSTEM-1: weight $=1.1$ ( standard deviation 0.07);

- SYSTEM-2: weight $=1.16$ (standard deviation 0.15).

These results show that the number of packets in conjunction with the direction of traffic can be reasonably used to split CPU consumption among guests. Concretely, 
let $\operatorname{Send} / \operatorname{Recv}\left(\operatorname{Dom}_{i}\right)$ denote packets sent/received by net-IDD to/from $\operatorname{Dom}_{i}$ and Send/Recv $($ netIDD) denote the total packets sent/received by net-IDD. Then, we define the weighted packet count per domain as weight $\times \operatorname{Send}\left(\operatorname{Dom}_{i}\right)+\operatorname{Recv}\left(\operatorname{Dom}_{i}\right)$, where weight is the ratio of CPU consumption in net-IDD for send versus receive paths. Similarly, we compute the weighted packet count for net-IDD: $w$ Count $(n e t I D D)$. Then we can use the fraction $w \operatorname{Count}\left(\operatorname{Dom}_{i}\right) / w \operatorname{Count}(n e t I D D)$ to charge CPU usage to $\operatorname{Dom}_{i}$.

In the remainder of this paper, we use this weighted count to compute the CPU overhead in net-IDD for network processing on behalf of different guest domains. This approach is also attractive because it comes with a compact, portable benchmark that derives the weight coefficient between send/receive paths automatically for different systems and different network device drivers. It has the further advantage of being general to a variety of device drivers and operating systems (e.g., individual device drivers may be hosted on a variety of operating systems) without requiring error-prone instrumentation. Of course, it has the disadvantage of not explicitly measuring CPU consumption but rather deriving it based on benchmarks of a particular hardware configuration. We feel that this trade off is inherent and that instrumenting all possible device driver/OS configurations is untenable for resource isolation. A variety of middleware tools face similar challenges, i.e., the inability to modify or directly instrument lower layers, making our approach attractive for alternate settings as well.

With this estimation of CPU utilization per guest, we now turn our attention to SEDFDC and ShareGuard.

\section{SEDF-DC: CPU Scheduler with Feedback}

Xen's reservation based CPU scheduler - SEDF (Simple Earliest Deadline First) takes its roots in the Atropos scheduler [8]. In SEDF, an administrator can specify the CPU share to be allocated per VM. However, there is no way to restrict the aggregate CPU consumed by a domain and by driver domains acting on its behalf. We have extended SEDF to accomplish this goal.

\subsection{Overview}

Our modified scheduler, SEDF-DC for SEDF-Debt Collector, periodically receives feedback from XenMon about the CPU consumed by IDDs for I/O processing on behalf of guest domains. Using this information, SEDF-DC constrains the CPU allocation to guest domains to meet the specified combined CPU usage limit.

For each domain $D o m_{i}$, SEDF takes as input a tuple $\left(s_{i}, p_{i}\right)$, where the slice $s_{i}$ and the period $p_{i}$ together represent the CPU share of $D o m_{i}: D o m_{i}$ will receive at least $s_{i}$ units of time in each period of length $p_{i}$. Such specifications are particularly convenient for dynamically adjusting CPU allocations: we can directly charge the CPU time consumed by IDDs for $D o m_{i}$ by decreasing $s_{i}$ appropriately. In CPU schedulers based on weights, one would need to continuously re-calculate weights of domains to achieve the same result.

We now describe SEDF-DC's operation, but limit our description only to places where SEDF-DC differs from SEDF. SEDF-DC maintains 3 queues: 
- $Q_{r}$ : the queue of runnable domains;

$-Q_{w}$ : the queue of domains that have exhausted their slice and are awaiting the next period;

- $Q_{b}$ : the queue of blocked domains.

A key concept in SEDF is deadlines. Intuitively, a deadline denotes the absolute time that a domain should have received its specified share of the CPU. Both $Q_{r}$ and $Q_{w}$ are sorted by deadlines, making the selection of the next domain to schedule a constant time operation.

Each domain $D_{i}$ 's deadline is initialized to $N O W+p_{i}$, where $N O W$ denotes the current time. Let $t$ denote the feedback interval (set to $500 \mathrm{~ms}$ in our current implementation). Let net-IDD be a driver domain with a networking device that is shared by $D_{\mathrm{om}}, \ldots, \mathrm{Dom}_{n}$. We will simplify the algorithm description (without loss of generality) by considering a single net-IDD. Using XenMon, we compute the CPU consumption $u s e d_{i}^{I D D}$ of net-IDD for network I/O processing on behalf of $D_{o m}$ during the latest $t$-ms interval and provide this information (for all domains) to SEDF-DC.

For each domain $D o m_{i}$, the scheduler tracks three values $\left(d_{i}, r_{i}, d e b t_{i}^{I D D}\right)$ :

- $d_{i}$ : domain's current deadline for CPU allocation, the time when the current period ends for domain $D_{o m}$.

- $r_{i}$ : domain's current remaining time for CPU allocation, the CPU time remaining to domain $D_{o m}$ within its current period.

- $\operatorname{debt}_{i}^{I D D}$ : CPU time consumed by $D_{0} m_{i}$ via the net-IDD's networking process-

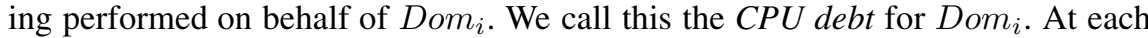
feedback interval, this value is incremented by $\operatorname{used}_{i}^{I D D}$ for the latest $t$-ms.

Note that the original SEDF scheduler only tracks $\left(d_{i}, r_{i}\right)$. The introduction of $\operatorname{debt}_{i}^{I D D}$ in the algorithm allows us to observe and enforce aggregate limits on $\operatorname{Dom}_{i}$ 's CPU utilization.

Let $a$ and $b$ be integer numbers and let us introduce the following function $\hat{a-b}$ as follows:

$$
a \hat{-} b= \begin{cases}0 & \text { if } a \leq b \\ a-b & \text { otherwise }\end{cases}
$$

We now describe the modified procedure for updating the queues $\left(Q_{r}, Q_{w}\right.$, and $\left.Q_{b}\right)$ on each invocation of SEDF-DC.

1. The time gotten $_{i}$ for which the current $D_{0} m_{i}$ has been running is deducted from $r_{i}: r_{i}=r_{i}-$ gotten $_{i}$

If $\operatorname{debt}_{i}^{I D D}>0$ then we attempt to charge $D_{0} m_{i}$ for its CPU debt by decreasing the remaining time of its CPU slice:

- if $d e b t_{i}^{I D D} \leq r_{i}$ then $r_{i}=r_{i}-d e b t_{i}^{I D D}$ and $d e b t_{i}^{I D D}=0$;

- if $d e b t_{i}^{I D D}>r_{i}$ then $\operatorname{debt}_{i}^{I D D}=d e b t_{i}^{I D D}-r_{i}$ and $r_{i}=0$.

2. If $r_{i}=0$, then $D o m_{i}$ is moved from $Q_{r}$ to $Q_{w}$, since $D o m_{i}$ has received its required $\mathrm{CPU}$ time in the current period.

3. For each domain $D_{o m}$ in $Q_{w}$, if $N O W \geq d_{k}$ then we perform the following updates: 
- $r_{k}$ is reset to $s_{k} \hat{-} \operatorname{debt}_{k}^{I D D}$;

- $\operatorname{debt}_{k}^{I D D}$ is decreased by $\min \left(s_{k}, \operatorname{debt}_{k}\right)$;

- the new deadline is set to $d_{k}+p_{k}$;

- If $r_{k}>0$ then $D o m_{k}$ is moved from $Q_{w}$ to $Q_{r}$.

4. The next timer interrupt is scheduled for $\min \left(d_{w}^{h}+p_{w}^{h}, d_{r}^{h}\right)$, where $\left(d_{w}^{h}, p_{w}^{h}\right)$ and $\left(d_{r}^{h}, p_{r}^{h}\right)$ denote the deadline and period of the domains that are respective heads of the $Q_{r}$ and $Q_{w}$ queues.

5. On an interrupt, the scheduler runs the head of $Q_{r}$. If $Q_{r}$ is empty, it selects the head of $Q_{w}$.

6. When domain $D o m_{k}$ in $Q_{b}$ is unblocked, we make the following updates:

- if $N O W<d_{k}$ then

- if $\operatorname{debt}_{k}^{I D D} \leq r_{k}$ then $r_{k}=r_{k}-\operatorname{debt}_{k}^{I D D}$, and $d e b t_{k}^{I D D}=0$, and $D_{o m}$ is moved from $Q_{b}$ to $Q_{r}$;

- if $d e b t_{k}^{I D D}>r_{k}$ then $\operatorname{debt}_{k}^{I D D}=\operatorname{debt}_{k}^{I D D}-r_{k}$ and $r_{k}=0$.

- if $N O W \geq d_{k}$ then we compute for how many periods $D o m_{k}$ was blocked. Since $D_{o m}$ was not runnable, this unused CPU time can be charged against its CPU debt:

$$
\begin{aligned}
\text { bl_periods } & =\operatorname{int}\left\{\frac{\left(N O W-d_{k}\right)}{p_{k}}\right\} \\
d e b t_{k}^{I D D} & =\operatorname{debt}_{k}^{I D D}-r_{k}-\left(b_{-} \text {periods } \times s_{k}\right)
\end{aligned}
$$

- $r_{k}$ is reset to $s_{k} \hat{-} \operatorname{debt}_{k}^{I D D}$. If $r_{k}>0$, then $D o m_{k}$ is moved from $Q_{b}$ to $Q_{r}$ and can be scheduled to receive the remaining $r_{k}$;

- $\operatorname{debt}_{k}^{I D D}$ is adjusted by $s_{k}: \operatorname{debt}_{k}^{I D D}=\operatorname{debt}_{k}^{I D D} \hat{-} s_{k}$;

- the new deadline is set to $d_{k}+p_{k}$

The SEDF-DC implementation described above might have bursty CPU allocation for domains hosting network-intensive applications, especially when a coarser granularity time interval $t$ is used for the scheduler feedback, e.g., $t=2 \mathrm{~s}$. It might happen that domain $D_{o m}$ will get zero allocation of CPU shares for several consecutive periods until the CPU debt time $d e b t_{i}^{I D D}$ is "repaid". To avoid this, we implemented an optimization to SEDF-DC that attempts to spread the CPU debt across multiple execution periods.

We compute the number of times period $p_{i}$ fits within a feedback interval - the intent is to spread the CPU debt of $D_{o m}$ across periods that happen during the feedback interval. We call this the CPU period frequency of domain $D_{o m}$ and denote it as period_freq $_{i}$ :

$$
\text { period_freq }_{i}=\operatorname{int}\left(\frac{t}{p_{i}}\right)
$$

If period $_{-} \mathrm{freq}_{i}>1$, then we can spread $d e b t_{i}^{I D D}$ across period_freq $q_{i}$ number of periods, where at each period the domain is charged for a fraction of its overall CPU debt:

$$
\text { spread_debt }_{i}=\operatorname{int}\left(\frac{\operatorname{debt}_{i}^{I D D}}{\text { period_freq }_{i}}\right)
$$

This optimized SEDF-DC algorithm supports more consistent and smoother CPU allocation to domains with network-intensive applications. 


\subsection{Evaluation}

In this section we evaluate SEDF-DC beginning with a simple setup to demonstrate the correctness of the scheduler and continue with a more complex scenario to illustrate SEDF-DC's feasibility for realistic workloads. All tests were conducted on single processor Pentium-IV machines running at 2.8-GHz.

In the first experiment, we have a single VM (Domain-1) configured to receive a maximum of $60 \%$ of the CPU; Domain-0 is entitled to the remaining $40 \%$. Domain-1 hosts a Web server, loaded using httperf [14] from another machine. We gradually increase the load and measure the resulting CPU utilization.

Figure 6a shows the results with the unmodified SEDF scheduler. We see that as the load increases, Domain-1 consumes almost all of its share of the CPU. Additionally, Domain-0 incurs an overhead of almost $35 \%$ at peak loads to serve Domain-1's traffic. Hence, while Domain-1 was entitled to receive $60 \%$ of the CPU, it had received a combined CPU share of $90 \%$ via additional I/O processing in Domain- 0 . We repeated the same experiment with SEDF-DC, with the results shown in Figure 6b We can see that SEDF-DC is able to enforce the desired behavior, keeping the combined CPU usage of Domain-1 bounded to $60 \%$.

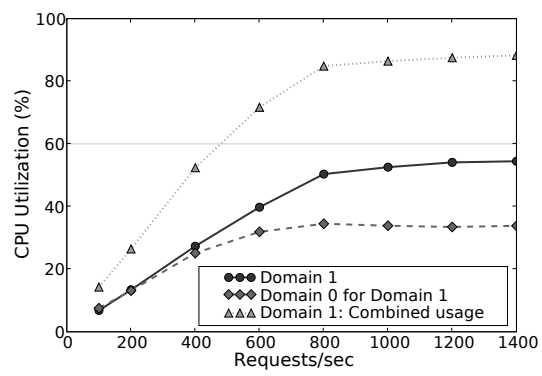

(a) With original SEDF

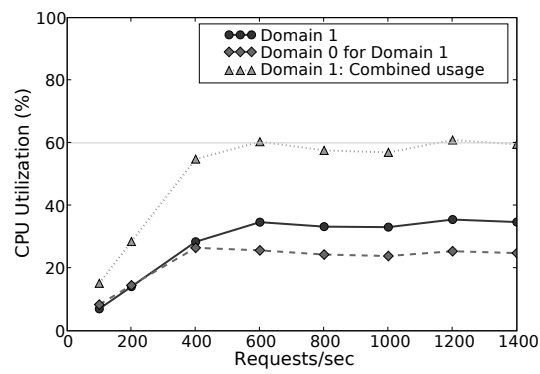

(b) With SEDF-DC

Fig. 6. Simple SEDF Benchmark

In practice, system configurations are likely to be more complicated: multiple VMs, each running a different service with different requirements; some VMs may be I/O intensive, others might be CPU intensive and so on. Our next experiment tries to evaluate SEDF and SEDF-DC under a more realistic setup.

For this experiment, we have two VMs (Domain-1 and Domain-2), each hosting a web-server. We configure both VMs and Domain- 0 to receive a maximum of $22 \%$ of the CPU. Any slack time in the system is consumed by CPU intensive tasks running in a third VM. Domain-1's web-server is served with requests for files of size $10 \mathrm{~KB}$ at 400 requests/second, while Domain-2's web-server is served with requests for files of size $100 \mathrm{~KB}$ at 200 requests/second. We chose these rates because they completely saturate Domain- 0 and demonstrate how CPU usage in Domain-0 may be divided between guest domains with different workload requirements. As before, we use httperf to generate client requests. Each run lasts 60 seconds. 
We first conduct the experiment with unmodified SEDF to establish the baseline. Figure $7 \mathrm{a}$ shows the throughput of the two web-servers as a function of time. We also measure the CPU utilization's of all the VMs, shown in 7b. Note that Domain-1 consumes all of its $22 \%$ available CPU cycles, while Domain- 2 consumes only about $15 \%$ of the CPU. Even more interesting is the split of Domain-0 CPU utilization across Domain-1 and Domain-2 as shown in Figure 7c For clarify, we summarize the experiment in Table 1. The first column shows the average values for the metrics over the entire run. Domain-1 uses an additional 9.6\% of CPU for I/O processing in Domain-0 (42\% of overall Domain-0 usage) while Domain-2 uses an additional $13.6 \%$ of CPU via Domain-0 (58\% of overall Domain-0 usage). Thus, the combined CPU utilization of Domains 1 and 2 (the sum of their individual CPU utilization and CPU overhead in Domain- 0 on their behalf) is $29.2 \%$ and $27.7 \%$ respectively.

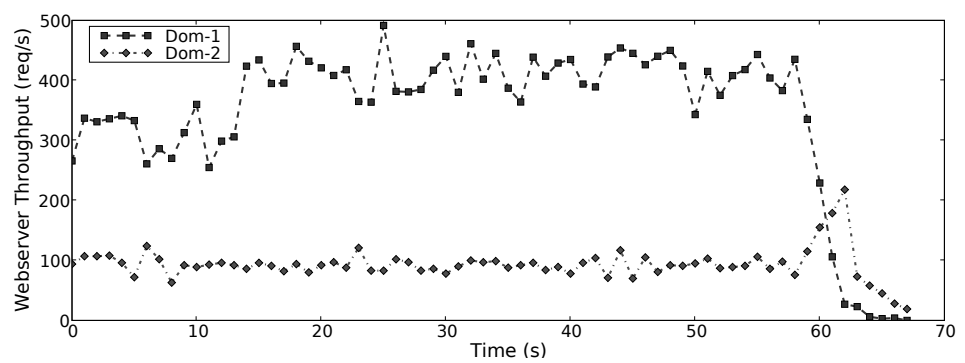

(a) Web-server Throughput

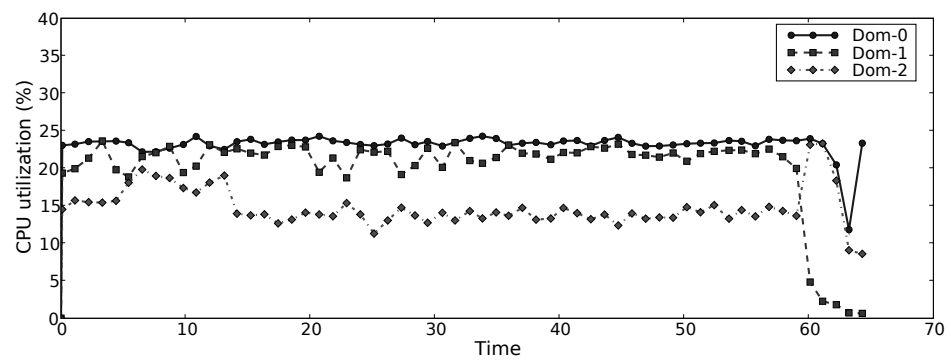

(b) CPU Utilization

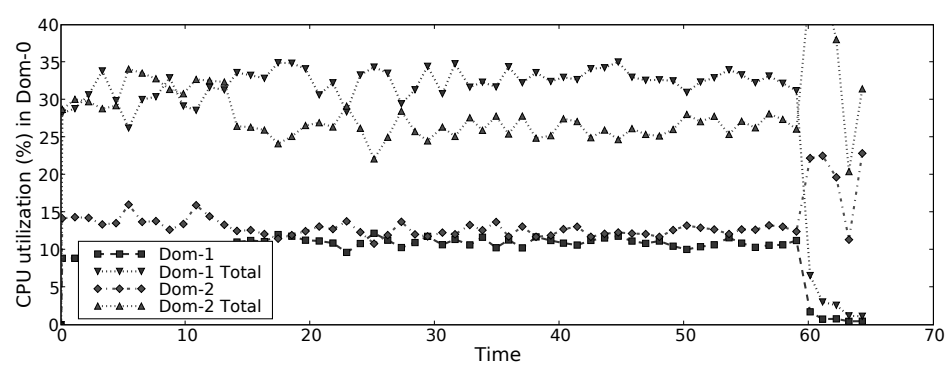

(c) CPU Utilization in Dom-0

Fig. 7. With original SEDF 
Table 1. SEDF-DC in action: metric values averaged over the duration of the run

\begin{tabular}{|l|c|c|}
\hline Metric & SEDF & SEDF-DC \\
\hline Dom-1 web-server Throughput & $348.06 \mathrm{req} / \mathrm{s}$ & $225.20 \mathrm{req} / \mathrm{s}$ \\
\hline Dom-2 web-server Throughput & $93.12 \mathrm{req} / \mathrm{s}$ & $69.53 \mathrm{req} / \mathrm{s}$ \\
\hline \hline Dom-1 CPU & $19.6 \%$ & $13.7 \%$ \\
\hline Dom-0 for Dom-1 & $9.6 \%$ & $7.7 \%$ \\
\hline Dom-1 Combined & $29.2 \%$ & $21.4 \%$ \\
\hline \hline Dom-2 CPU & $14.5 \%$ & $10.9 \%$ \\
\hline Dom-0 for Dom-2 & $13.2 \%$ & $10.6 \%$ \\
\hline Dom-2 Combined & $27.7 \%$ & $21.5 \%$ \\
\hline
\end{tabular}

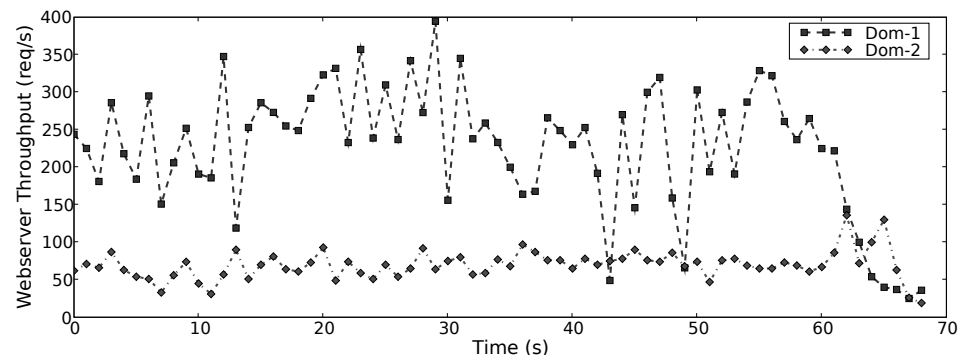

(a) Web-server Throughnut

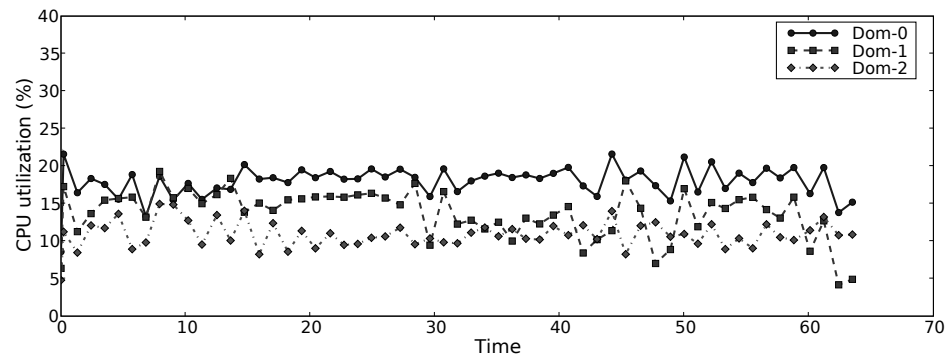

(b) CPU Utilization

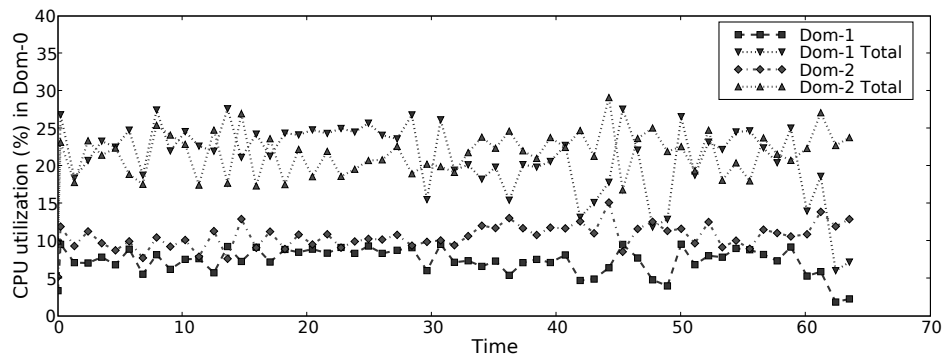

(c) CPU Utilization in Dom- 0

Fig. 8. With SEDF-DC 
We then investigate whether we can limit the system-wide CPU usage of Domain-1 and Domain-2 to their 22\% CPU share using SEDF-DC. Figure 8 shows the results of this experiment. Recall the operation of SEDF-DC: it computes the debt of a VM (work done by the IDD - in this case Domain-0 - on its behalf), and incrementally charges it back to the appropriate VM. This is clearly visible in Figure $8 \mathrm{c}$ the combined utilization's of both Domain-1 and Domain-2 hover around 22\% for the duration of the experiment. The oscillations result from discretization in the way we charge back debt.

Controlling combined CPU utilization for Domain- 1 and 2 does impact the web servers' achievable throughput. Since the combined CPU usage of Domain-1 and 2 is limited to $22 \%$ under SEDF-DC_-versus the uncontrolled values of $29.2 \%$ and $27.7 \%$ under the original SEDF scheduler-there is a drop in throughput as shown in Figure $8 \mathrm{a}$. The second column of Table 1 gives the average throughput values over the run for a more concise comparison.

While SEDF-DC is capable of limiting the combined CPU usage across guest domains, it does not explicitly control CPU usage in a driver domain. Note that the split of the CPU utilization in Domain-0 for Domain-1 and Domain-2 is still unequal. Domain-1 is using $7.7 \%$ of CPU via Domain-0 (42\% of overall Domain-0 usage) while Domain2 is using $10.6 \%$ of CPU via Domain-0 (58\% of overall Domain-0 usage). We turn our attention to controlling per-domain IDD utilization using ShareGuard in the next section.

\section{ShareGuard}

In the current Xen implementation, a driver domain does not control the amount of CPU it consumes for I/O processing on behalf of different guest domains. This lack of control may significantly impact the performance of network services. Such control is also required to enable SEDF-DC to enforce aggregate CPU usage limits. In this section we describe ShareGuard: a control mechanism to solve this problem.

\subsection{Overview}

ShareGuard is a control mechanism to enforce a specified limit on CPU time consumed by an IDD for I/O processing on behalf of a particular guest domain. ShareGuard periodically polls XenMon for CPU time consumed by IDDs, and if a guest domain's CPU usage is above the specified limit, then ShareGuard stops network traffic to/from the corresponding guest domain.

Let the CPU requirement of net-IDD be specified by a pair $\left(s^{I D D}, p^{I D D}\right)$, meaning that net-IDD will receive a CPU allocation of at least $s^{I D D}$ time units in each period of length $p^{I D D}$ units (the time unit is typically milli-seconds). In other words, this specification is bounding CPU consumption of net-IDD over time to $C P U \operatorname{share}^{I D D}=$ $\frac{s^{I D D}}{p^{I D D}}$. Let limit $_{i}^{I D D}$ specify a fraction of CPU time in net-IDD available for network processing on behalf of $D_{o m}$ such that limit $_{i}^{I D D}<C P U$ share ${ }^{I D D}$. If such a limit is not set then $D_{o m}$ is entitled to unlimited I/O processing in net-IDD. Let $t$ be the time period ShareGuard uses to evaluate current CPU usage in net-IDD and perform decision making. In the current implementation of ShareGuard, we use $t=500 \mathrm{~ms}$. 
Using XenMon, ShareGuard collects information on CPU usage by net-IDD at every feedback interval, and computes the fraction of overall CPU time used by net-IDD for networking processing on behalf of $\operatorname{Dom}_{i}(1 \leq i \leq n)$ during the latest $t$ interval. Let us denote this fraction as $u \operatorname{sed}_{i}^{I D D}$. In each time interval $t$, ShareGuard determines the validity of the condition: $\operatorname{used}_{i}^{I D D} \leq \operatorname{limit}_{i}^{I D D}$. If this condition is violated, then $\operatorname{Dom}_{i}$ has exhausted its CPU share for network traffic processing in net-IDD. At this point, ShareGuard applies appropriate defensive actions for the next time interval $t^{\text {def }}$, where

$$
t^{d e f}=t \times \operatorname{int}\left(\frac{u s e d_{i}^{I D D}+1}{\operatorname{limit}_{i}^{I D D}}\right)
$$

ShareGuard performs the following defensive actions:

- Stop accepting incoming traffic to a domain: Since our net-IDDs run Linux, we use Linux's routing and traffic control mechanisms [15] to drop/reject traffic destined for a particular domain. In particular, we use iptables [16] - they are easily scriptable and configurable from user space. Similar techniques can be applied in other operating systems that may serve as wrappers for other legacy device drivers.

- Stop processing outgoing traffic from a domain: As in the previous case, we can use iptables to drop packets being transmitted from a domain. However, this will still incur substantial overhead in the IDD because iptables will only process the packet once it has traversed the network stack of the IDD. Ideally we want to drop the packet before it even enters the IDD to limit processing overhead.

One approach would be to enforce iptables filtering within the guest domain. However, ShareGuard does not assume any cooperation from guests so we reject this option. However, we still have an attractive control point within the net-IDD where packets can be dropped before entering the net-IDDs network stack: the netback driver (see Figure 3). ShareGuard sends a notification to netback identifying the target domain and the required action (drop or forward). This is akin to setting iptables rules, except that these rules will be applied within netback.

Whenever netback receives an outbound packet from a domain, it will determine if there are any rules applicable to this domain. If so, it will take the specified action. This is both lightweight (in terms of overhead incurred by IDD) and flexible (in terms of control exercised by IDD).

After time interval $t^{\text {def }}$, ShareGuard restores normal functionality in net-IDD with respect to network traffic to/from domain $\operatorname{Dom}_{i}$.

\subsection{Evaluation}

To evaluate the effectiveness of ShareGuard in isolating total domain CPU consumption, we ran the following experimental configuration. Three virtual machines run on the same physical hardware. Domain-1 and Domain-2 host web servers that support business critical services. These services have well-defined expectations for their throughput and response time. The CPU shares for these domains are set to meet these expectations. Domain-3 hosts a batch application that does some computation and performs 
occasional bulk data transfers. This VM supports a less important application that is not time sensitive, but needs to complete its job eventually.

In our first experiment, we observe overall performance of these three services to quantify the degree of performance isolation ShareGuard can deliver. We configure a dual-processor machine as follows: Domain-0 runs on a separate processor and set to consume at most $60 \%$ of the CPU. The second CPU hosts three VMs: Domain-1 and Domain-2 run web servers (serving $10 \mathrm{~KB}$ and $100 \mathrm{~KB}$ files respectively), and Domain-3 occasionally does a bulk file transfer. All these VMs have equal share of the second CPU, 33\% each. In this initial experiment, we do not enable ShareGuard to demonstrate baseline performance characteristics. The experiments were conducted over a gigabit network, so our experiments are not network limited. In this experiment, we start a benchmark that loads web servers in Domain-1 and Domain-2 from two

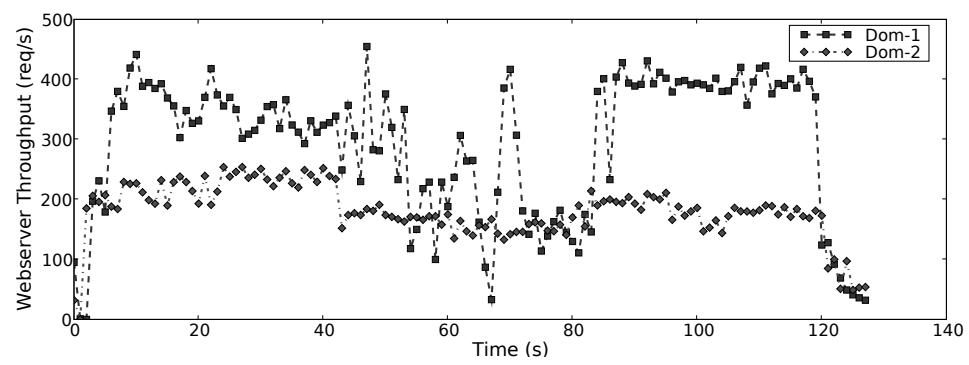

(a) Web server Throughput

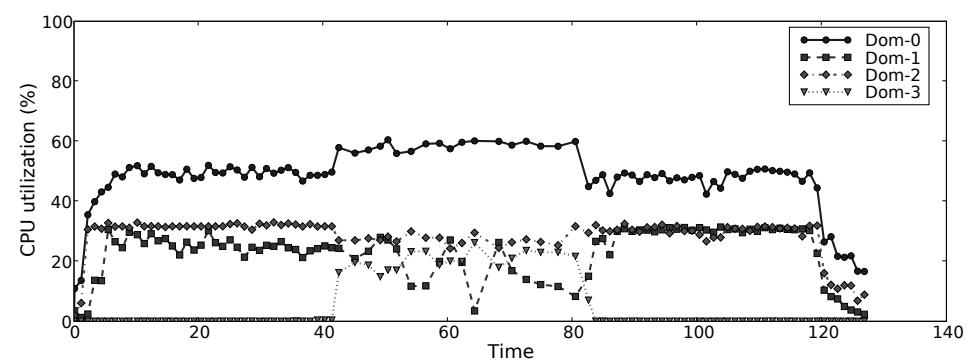

(b) CPU Utilization

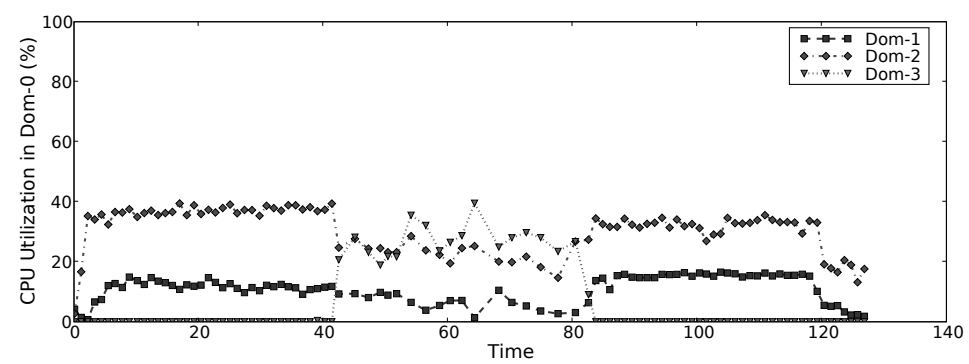

(c) CPU Utilization in Dom-0

Fig. 9. Without ShareGuard 
separate machines using httperf for two minutes. Forty seconds into the benchmark, Domain-3 initiates a bulk-file transfer that lasts for 40 seconds.

Figure 9 shows the results as a function of time. We can clearly see the adverse impact of Domain-3's workload on both web servers' throughput (Figure 9a). Considering the split of CPU utilization in Domain-0 for the corresponding interval (Figure 9c), we find that Domain-3 uses between $20 \%$ to $30 \%$ of CPU for I/O processing in Domain0 leaving insufficient CPU resources for I/O processing on behalf of Domain-1 and Domain-2.

The first column in Table 2 provides a summary of average metric values for the baseline case where Domain-1 and Domain-2 meet their performance expectations and deliver expected web server throughput. These metrics reflect Domain-1 and Domain-2 performance when there is no competing I/O traffic issued by Domain-3 in the experiment. Note that in this case the combined CPU utilization in Domain-0 for I/O processing by Domain- 1 and Domain- 2 is about $50 \%$. Since Domain- 0 is entitled to $60 \%$ of the CPU, this means that there is about $10 \% \mathrm{CPU}$ available for additional I/O processing in Domain-0.

Table 2. ShareGuard at work: metric values are averaged over the middle 40 second segment of the runs

\begin{tabular}{|l|c|c|c|}
\hline Metric & Baseline & Without ShareGuard & With ShareGuard \\
\hline Dom-1 Web server & 329.85 & 236.8 & 321.13 \\
\hline Dom-2 Web server & 231.49 & 166.67 & 211.88 \\
\hline \hline Dom-0 for Dom-1 & 11.55 & 7.26 & 11.9 \\
\hline Dom-0 for Dom-2 & 37.41 & 23.9 & 34.1 \\
\hline Dom-0 for Dom-3 & N/A & 23.92 & 4.42 \\
\hline
\end{tabular}

The average metric values for this experiment (without ShareGuard) over the middle 40 second segment (where Domain-1, Domain-2, and Domain-3 all compete for CPU processing in Domain-0) are summarized in the second column of Table 2. Domain-3 gets $23.92 \%$ of CPU for I/O processing in Domain-0, squeezing in the CPU share available for Domain-1's and Domain-2's I/O processing. As a result, there is a significant decrease in achievable web server throughput: both web servers are delivering only $72 \%$ of their expected baseline capacity.

This example clearly indicates the impact of not controlling IDD CPU consumption by different guest domains. The question is whether ShareGuard can alleviate this problem. We repeat the experiment with ShareGuard enabled, and configure ShareGuard to limit the CPU consumption for Domain-3 in Domain-0 to 5\%. Figure 10 shows the results.

Recall ShareGuard's operation: every $500 \mathrm{~ms}$ it evaluates CPU usage in the IDD; if a VM is violating its CPU share, it turns off all traffic processing for that VM for some time. We compute this duration such that over that interval, the average CPU utilization of the VM within the IDD will comply with the specification. This mode of operation is clearly visible in Figure 10c. We had directed ShareGuard to restrict Domain-3's consumption in Domain-0 to 5\%. At $t=40 \mathrm{~s}$, ShareGuard detected that 
Domain-3 had consumed almost 30\% CPU in Domain-0. Accordingly, it disables traffic processing for Domain-3 for the next 2.5 seconds, such that the average utilization over this 3 second window drops to $5 \%$. This pattern subsequently repeats ensuring that the isolation guarantee is maintained through the entire run.

Comparing Figure $9 \mathrm{c}$ and $10 \mathrm{c}$ we see that with ShareGuard, Domain-1 and Domain2 obtain more uniform service in Domain-0 even in the presence of Domain-3's workload. This is also visible in the CPU utilization (see Figure 10b). Finally, observe that the web-server throughput for Domain-1 and Domain-2 improve significantly under ShareGuard: both web servers deliver the expected throughput.

The third column in Table 2 provides a summary of average metric values over the middle 40 second segment with ShareGuard enabled. As we can see, CPU consumption by Domain-1 and Domain-2, as well as web server throughput are similar to the baseline

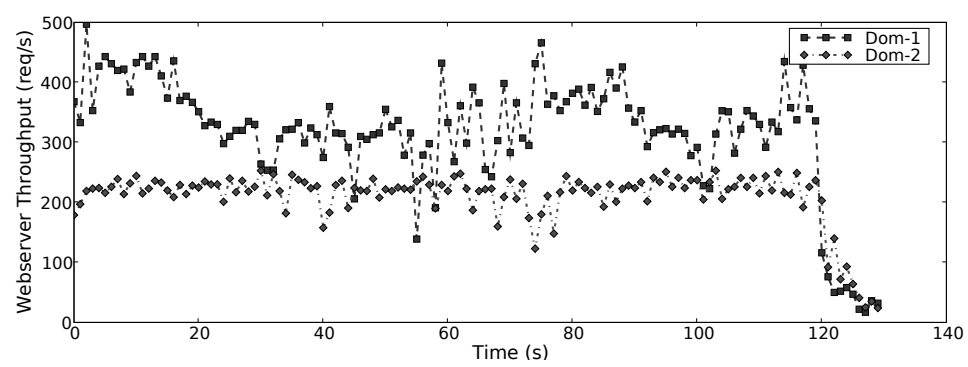

(a) Web server Throughput

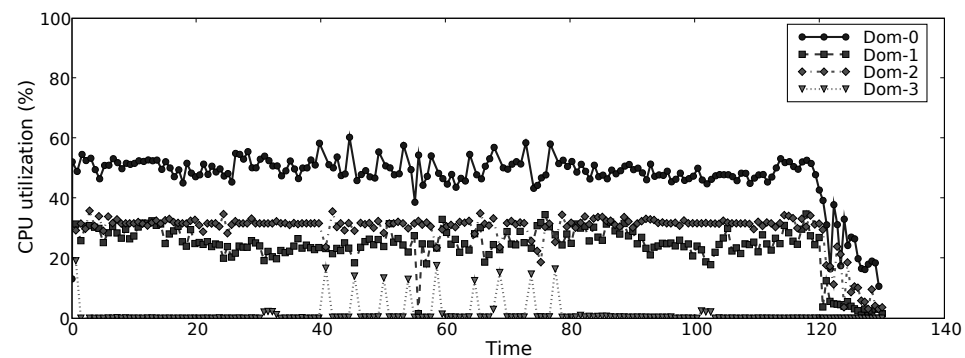

(b) CPU Utilization

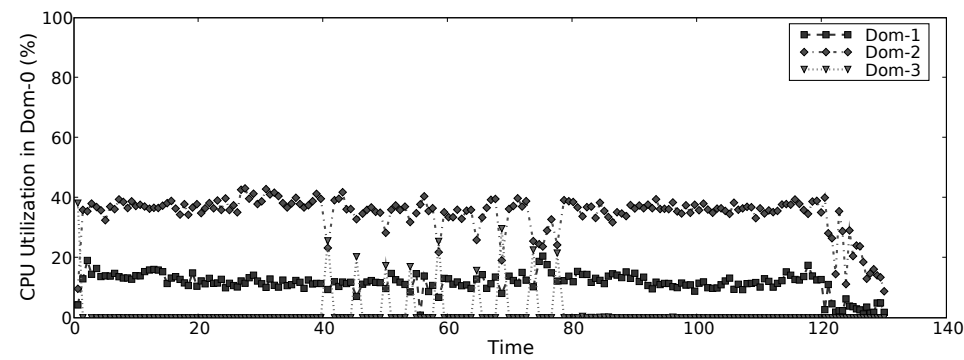

(c) CPU Utilization in Dom-0

Fig. 10. With ShareGuard 
case. Web server performance does not degrade in presence of the bulk data transfer in Domain-3 because CPU processing in the IDD on behalf of Domain-3 is controlled by ShareGuard.

\section{Discussion}

All three of the components discussed in this play important, complementary tasks in enforcing performance isolation. Both SEDF-DC and ShareGuard depend on XenMon for detailed CPU utilization information. While ShareGuard is only relevant for workloads involving network I/O, SEDF-DC is agnostic to the choice of workloads - it only depends on accurate feedback on CPU utilization from XenMon.

However, SEDF-DC can only enforce guarantees on the aggregate CPU consumption of a guest and its IDD - it does not consider fair allocation of the driver domain's finite CPU resources. ShareGuard can be used to enforce such limits for networking workloads. Further, ShareGuard works irrespective of the choice of CPU scheduler. An artifact of Xen's current CPU schedulers in Xen is that SEDF-DC only works for single processor systems. ShareGuard, however, supports multi-processor systems as well. We expect that this limitation will be removed with future releases of Xen.

Finally, ShareGuard is more intrusive in the sense that it actively blocks a VM's traffic. In comparison, SEDF-DC is more passive and transparent. Also, as shown in Section 5. CPU allocation in ShareGuard is more bursty than in SEDF-DC (compare Figures $8 \mathrm{c}$ and $10 \mathrm{c}$. All this underscores the fact that while on its own no single mechanism is perfect, working together they form a complete system.

\section{Related Work}

The problem of resource isolation is as old as time sharing systems. Most of the previous work in this area has focused on resource isolation between processes in an operating system or users on a single machine. In these systems, scheduling and resource management primitives do not extend to the execution of significant parts of kernel code. An application has no control over the consumption of many system resources that the kernel consumes on behalf of the application.

Consider network-intensive applications: most of the processing is typically done in the kernel and the kernel generally does not control or properly account for resources consumed during the processing of network traffic. The techniques used in ShareGuard have been inspired by earlier work addressing this problem with respect to receive livelocks in interrupt based networking subsystems. Mogul et al. [17] restrict the amount of I/O processing that the kernel does on behalf of user processes. In Lazy Receiver Processing [9] (LRP), the system uses better accounting information (such as hardware support for identifying which process an incoming packet is destined to) to improve resource isolation, e.g., such that packet processing on behalf of one process does not adversely affect the resource available to other processes.

Some of the ideas motivating LRP were extended to Resource Containers [12]. A resource container is an operating system abstraction to account for all system resources consumed by an activity, where an activity might span multiple processes. Resource 
Containers separate the notion of resource principal from threads or processes and provide support for fine-grained resource management in operating systems. This distinction between a protection domain and a resource principal is also visible in Xen's new I/O model: a VM (the protection domain) may request service from several different IDDs, therefore the tracking of its resource usage needs to span across executions of all these domains.

One limitation of Resource Containers is that they only work for single processor systems. There does not seem to be any straightforward way of extending the notion of an activity to span multiple processors. This is further complicated by the fact that in most operating systems, each CPU is scheduled independently. SEDF-DC scheduler suffers from the same limitation. However, ShareGuard is both scheduler agnostic and it fully supports multi-processor systems.

The problem of performance isolation has been actively addressed by multimedia systems [8 18]. The Nemesis operating system [8] was designed to provide guaranteed quality of service (QoS) to applications. Nemesis aims to prevent QoS crosstalk that can occur when the operating system kernel (or a shared server) performs a significant amount of work on behalf of a number of applications. One key way in which Nemesis supports this isolation is by having applications execute as many of their own tasks as possible. Since a large proportion of the code executed on behalf of an application in a traditional operating system requires no additional privileges and does not, therefore, need to execute in a separate protection domain, the Nemesis operating system moves the majority of operating system services into the application itself, leading to a vertically structured operating system. QoS crosstalk can also occur when there is contention for physical resources, and applications do not have guaranteed access to the resources. Nemesis provides explicit low-level resource guarantees or reservations to applications. This is not limited simply to CPU: all resources including disks [19], network interfaces [20], and physical memory [21] - are treated in the same way.

The networking architecture of Nemesis still has some problems related to the charging of CPU time to applications. When the device driver transmits packets for an application, used CPU time is not charged to the application but to the device driver. Also, the handling of incoming packets before de-multiplexing it to the receiving application is charged to the device driver. We observe the same problem in the context of Xen VMM and the network driver domains, and suggest possible solution to this problem.

Exokernel [22] and Denali [23] provide resource management systems similar to vertically structured operating systems. The design goal for Exokernel was to separate protection from management. In this architecture, a minimal kernel - called Exokernel - securely multiplexes available hardware resources. It differs from the VMM approach in that it exports hardware resources rather than emulates them. VMMs have served as the foundation of several "security kernels" [24|25 26|27]. Denali differs from these efforts in that it aims to provide scalability as well as isolation for untrusted code, but it does not provide any specialized for performance isolation.

Most of the earlier work on VMMs focused on pursuing OS support for isolating untrusted code as a primary goal. While there is significant work on resource management in traditional operating systems, relatively less work has been performed in the context of virtual machines. Waldspurger [28] considers the problem of allocating 
memory across virtual machines; other systems such as Denali [23], HP SoftUDC [2] and Planetlab vServers [29] have also touched on some of these issues. Our work takes another step towards a general framework for strict resource isolation in virtual machines by considering the auxiliary work done on behalf of a guest in privileged or driver domains.

\section{Conclusion and Future Work}

Virtualization is fast becoming a commercially viable alternative for increasing system utilization. But from a customer perspective, virtualization cannot succeed without providing appropriate resource and performance isolation guarantees. In this work, we have proposed two mechanisms - SEDF-DC and ShareGuard - that improve CPU and network resource isolation in Xen. We demonstrated how these mechanisms enable new policies to ensure performance isolation under a variety of configurations and workloads.

For future work, we plan to extend these mechanisms to support other resources such as disk I/O and memory. Work is also underway on a hierarchical CPU scheduler for Xen: currently Xen ships with two CPU schedulers, but the choice of scheduler has to be fixed at boot time. We expect that in the future, many more CPU schedulers will become available (SEDF-DC being among the first), and that having a hierarchical scheduler that allows the use of different schedulers for different domains depending on the kinds of applications and workloads that need to be supported will enable more efficient resource utilization.

We believe that performance isolation requires appropriate resource allocation policies. Thus, another area for future investigation is policies for efficient capacity planning and workload management.

\section{References}

1. Barham, P., Dragovic, B., Fraser, K., Hand, S., Harris, T., Ho, A., Neugebauer, R., Pratt, I., Warfield, A.: Xen and the art of virtualization. In: Proc. of the 19th ACM SOSP, New York, NY (2003)

2. Kallahalla, M., Uysal, M., Swaminathan, R., Lowell, D.E., Wray, M., Christian, T., Edwards, N., Dalton, C.I., Gittler, F.: SoftUDC: A software based data center for utility computing. IEEE Computer (2004)

3. The Oceano Project. http://www.research.ibm.com/oceanoproject/ index.html Last accessed 1/17/2006.

4. Fraser, K., Hand, S., Neugebauer, R., Pratt, I., Warfield, A., Williamson, M.: Reconstructing I/O. Technical Report UCAM-CL-TR-596, University of Cambridge (2005)

5. Cherkasova, L., Gardner, R.: Measuring CPU Overhead for I/O Processing in the Xen Virtual Machine Monitor. In: Proc. of USENIX Annual Technical Conference. (2005)

6. Chase, J.S., Levy, H.M., Feeley, M.J., Lazowska, E.D.: Sharing and protection in a singleaddress-space operating system. ACM Trans. Comput. Syst. 12(4) (1994) 271-307

7. Jones, M.B., Leach, P.J., Draves, R.P., J. S., .I.B.: Modular real-time resource management in the Rialto operating system. In: Proc. of the 5th HotOS, Washington, DC, USA, IEEE Computer Society (1995) 12 
8. Leslie, I.M., McAuley, D., Black, R., Roscoe, T., Barham, P.T., Evers, D., Fairbairns, R., Hyden, E.: The design and implementation of an operating system to support distributed multimedia applications. IEEE Journal of Selected Areas in Communications 14(7) (1996)

9. Druschel, P., Banga, G.: Lazy receiver processing (LRP): a network subsystem architecture for server systems. In: Proc. of the second USENIX OSDI. (1996) 261-275

10. Bruno, J., Gabber, E., Ozden, B., Silberschatz, A.: The Eclipse Operating System: Providing Quality of Service via Reservation Domains. USENIX Annual Technical Conference (1998)

11. Verghese, B., Gupta, A., Rosenblum, M.: Performance isolation: sharing and isolation in shared-memory multiprocessors. In: Proc. of the 8th International Conference on Architectural Support for Programming Languages and Operating Systems, New York, NY, USA, ACM Press (1998) 181-192

12. Banga, G., Druschel, P., Mogul, J.C.: Resource Containers: a New Facility for Resource Management in Server Systems. In: Proc. of the third USENIX OSDI, New Orleans, Louisiana (1999)

13. Gupta, D., Gardner, R., Cherkasova, L.: XenMon: QoS Monitoring and Performance Profiling Tool. Technical report, HPL-2005-187 (2005)

14. Httperf. http://www.hpl.hp.com/research/linux/httperf/

15. http://www. lartc.org/howto/: Last accessed 04/02/2006.

16. http: / / www.netfilter.org: Last accessed 04/02/2006.

17. Mogul, J.C., Ramakrishnan, K.K.: Eliminating receive livelock in an interrupt-driven kernel. ACM Trans. Comput. Syst. 15(3) (1997)

18. Yuan, W., Nahrstedt, K.: Energy-efficient soft real-time cpu scheduling for mobile multimedia systems. In: Proc. of the 19th SOSP, New York, NY, USA, ACM Press (2003) 149-163

19. Barham, P.: A Fresh Approach to File System Quality of Service. In Proc. of NOSSDAV (1998)

20. Black, R., Barham, P., Donnelly, A., Stratford, N.: Protocol Implementation in a Vertically Structured Operating System. In: Proc. of IEEE Conference on Computer Networks. (1997)

21. Hand, S.M.: Self-paging in the Nemesis operating system. In: Proc. of the third USENIX OSDI, Berkeley, CA, USA, USENIX Association (1999) 73-86

22. Engler, D.R., Kaashoek, M.F., J. O'Toole, J.: Exokernel: an operating system architecture for application-level resource management. In: Proc. of the 15th ACM SOSP, New York, NY, USA, ACM Press (1995) 251-266

23. Whitaker, A., Shaw, M., Gribble, S.D.: Scale and performance in the Denali isolation kernel. In: Proc. of the 5th USENIX OSDI, Boston, MA (2002)

24. Karger, P.A.: A retrospective of the VAX VMM security kernel. IEEE Trans, on Software Engineering (1991)

25. Meushaw, R., Simard, D.: NetTop: Commercial Technology in high assurance applications. (2005)

26. Bugnion, E., Devine, S., Rosenblum, M.: Disco: running commodity operating systems on scalable multiprocessors. In: Proc. of the 16th ACM SOSP, New York, NY, USA, ACM Press (1997) 143-156

27. Creasy, R.J.: The origin of the VM/370 time-sharing system. IBM Journal of Research and Development (1982)

28. Waldspurger, C.A.: Memory resource management in VMware ESX server. In: Proc. of the 5th USENIX OSDI. (2002)

29. Chun, B., Culler, D., Roscoe, T., Bavier, A., Peterson, L., Wawrzoniak, M., Bowman, M.: PlanetLab: an overlay testbed for broad-coverage services. SIGCOMM Comput. Commun. Rev. 33(3) (2003) 3-12 